\title{
Pour une construction interdisciplinaire du concept de milieu urbain durable
}

\author{
Nicole Mathieu \\ Géographe, UMR Ladyss, CNRS/Université de Paris 1, 2 rue Valette, 75005 Paris, France
}

\author{
Mots-clés : \\ développement \\ durable ; \\ ville durable ; \\ interdisciplinarité ; \\ mode d'habiter ; \\ milieux
}

\section{Keywords: \\ sustainable development; sustainable city; interdisciplinarity; inhabitance; "mode d'habiter"; milieu}

\begin{abstract}
Résumé - Ce texte théorique est délibérément orienté vers la mise en œuvre d'une pratique scientifique correspondant aux exigences de la notion de développement durable. Transversal, il tente une lecture de ce qui est dit et fait au nom du développement urbain durable, tant dans le champ du politique que dans celui du rapport des sciences sociales au politique. Il est aussi méthodologique dans la mesure où la démarche interdisciplinaire proposée pour instruire cet objet complexe de l'utopie du développement urbain durable en action implique le recours à l'échelle de l'habitant et à la compréhension de la ville comme un ensemble de milieux urbains. Il appelle à une mobilisation conjointe des systèmes d'information géographique et de la modélisation, mais aussi des méthodes anthropologiques sans lesquelles il est impossible d'évaluer les possibilités et les limites de la mise en œuvre d'une politique par nature complexe, puisqu'elle doit concilier des enjeux contradictoires.
\end{abstract}

\begin{abstract}
Call for building the interdisciplinary concept of sustainable urban milieu. Taking Sartre's philosophy as a starting point, our paper is deliberately both theoretical and action oriented. It aims to promote a way to develop the adequate scientific practices which the sustainable development utopia requires. To this end, it is written as a manifesto designed to stimulate debates and controversies. Following a critical review of all that is being said and done in the name of sustainable development in the political sphere as well as in the relationships social sciences entertain with the political sphere, this paper is a call to consider rigorously the difficulties and even the impossibilities of moving "from the utopia to the concepts". Introducing and articulating the almost contradictory dimensions of sustainability in real urban territories demands that a relevant method be developed to investigate this complex object. We propose the concept of "socially sustainable milieu" to try and reach this objective which requires the interdisciplinary linking of physical and social sciences. GIS and modelling need to be associated with anthropological methods. Most of all, the paper underscores the need to set "inhabitants" at the heart of the scientific project, thus reversing the usual focus and starting with an investigation of the way each individual reconciles the "three pillars" of sustainable development and builds his/her relationship with sustainable urban milieus. This may help rise above the still top-down views of governance, thus enabling scientists to contribute to developing the political sustainable utopia in real sites and situations.
\end{abstract}

\section{Introduction}

$2005^{1}$ est l'année Sartre et ce texte se voudrait «sartrien », non pas pour rendre hommage à ce philosophe, mais pour montrer qu'il inspire encore un avenir au-delà de son historiographie. Car, face à la question

\footnotetext{
Auteur correspondant : mathieu@univ-paris1.fr

${ }^{1}$ Ce texte est issu d'une communication présentée au colloque «Développement urbain durable, gestion des ressources et gouvernance », organisé par l'Observatoire universitaire de la ville et du développement durable (Université de Lausanne, 21-23 septembre 2005), communication que l'on pourra trouver dans le CD-Rom des actes du colloque (Mathieu et Cohen, 2005).
}

d'Olivier Godard (2001) : « Pourquoi donc les chercheurs devraient-ils, en tant que chercheurs et pas en tant que citoyens, se sentir concernés par le développement durable? », quelques « mots » de Sartre pourraient être un tremplin pour une réponse, certes personnelle, mais que l'on aimerait voir devenir collective. Ainsi ceux entendus cités au colloque de Cerisy-la-Salle ${ }^{2}$ : «Tout commandement dicté par une puissance extérieure doit être

\footnotetext{
2 «Jean-Paul Sartre : écriture et engagement », colloque organisé par le Centre culturel international de Cerisy-la-Salle (20-30 juillet 2005), où le point fut fait sur les perspectives que l'œuvre sartrienne ouvre au début du XXI $\mathrm{e}^{\mathrm{e}}$ siècle, en particulier sur le rapport entre recherche, écriture et engagement.
} 
délégitimé» (exposé de Paolo Tamassia) ; «L'histoire n'est pas un rapport au passé mais un rapport au futur » (citation reprise par Pierre Verstraeten); «C'est l'intensité de l'acte qui exprime l'intentionnalité morale » (par Gérard Wormser)...

C'est cette entreprise de construction « libre » d'un point de vue théorique qui trouve sa force dans ce que Sartre appelle une "intentionnalité morale » (Wormser, 2005) que l'on voudrait tenter ici. Écrit à la façon d'un manifeste, ce texte a pour objectif d'expliciter les conditions d'un mode de connaissance répondant à la complexité de l'utopie politique $^{3}$. D'ailleurs, ne faut-il pas être à la fois dans la position du philosophe tel que Sartre l'entend : une pensée libre en acte, et dans celle d'un scientifique soucieux de ses rapports avec la philosophie (Legay et Schmid, 2004), pour s'engager dans la réflexion sur ce que signifie «vers une ville socialement durable » et désirer redéfinir le lien entre le politique et le scientifique?

\section{Les conditions d'une radicalité scientifique : prendre l'utopie politique à la lettre pour produire un nouveau mode de connaissance}

Sans refaire l'état de l'art qui introduit la publication de La Ville durable, du politique au scientifique (Mathieu et Guermond, 2005b), il me faut reconnaître qu'il m'a permis une mise à distance de ce qui est dit et fait au nom du développement urbain durable, tant dans le champ du politique que dans celui du rapport des sciences sociales au politique; il m'a permis par là même de construire la position théorique autonome que je soumets ici à discussion. En effet, ce n'est qu'après avoir revisité les usages du terme de «développement urbain durable ${ }^{4}$ »-depuis son apparition en catégorie de pensée et d'action (Jollivet, 1992) recouvrant les catégories antérieures comme celles d'aménagement urbain, d'urbanisme, voire de « renouvellement urbain » ou de "régénération urbaine »... que j'ai pris conscience d'appartenir à un courant de recherche abordant la question urbaine sous un angle radicalement nouveau. C'est là, en fait, le fruit d'une longue réflexion partant de mes recherches sur le rural ${ }^{5}$.

\footnotetext{
${ }^{3}$ Cf. Mathieu et Guermond (2005a).

4 Et bien sûr des expressions «ville durable», "ville soutenable » ou... « insoutenable» (cf. dans ce numéro le texte de Joëlle Salomon Cavin).

${ }^{5}$ Ce courant, c'est celui qui a porté la « longue marche » que les sciences rurales ont entreprises pour passer « du rural à l'environnement » et poser «la question de la nature aujourd'hui » (Mathieu et Jollivet, 1989). C'est aussi celui qui, prolongeant cette ouverture, a entrepris de procéder à l'évaluation des acquis théoriques et méthodologiques des recherches des ruralistes qui se voulaient des "passeurs de frontières » entre «sciences de la nature » et «sciences de la société »
}

Le passage à l'urbain illustre la pertinence et la portée générale de la démarche mise en œuvre dans celles-ci lorsque l'on s'inscrit dans une perspective de développement durable .

L'état des lieux des recherches sur la ville mises en relation avec l'usage de la notion de durabilité partait d'une interrogation : l'irruption de la question du développement durable avait-elle « renouvelé » en profondeur les conceptions du rapport entre la connaissance et l'action? À première vue, du fait même de leur orientation vers la question toujours récurrente de la ville de demain ${ }^{7}$, les politiques comme les scientifiques ont du mal à sortir de schémas d'analyse antérieurs. Même quand le Plan Urbanisme Construction Architecture considère «que les modèles issus de la ville classique ne peuvent plus être uniformément convoqués pour les interventions urbaines » (Dubois-Taine, 1998, p. 5), la recherche des figures de « la ville émergente» ne prend de sens que pour déboucher sur des « constats pour renouveler les lignes d'action publique». On reste dans une démarche top-down. L'idée qu' «une vision renouvelée des territoires » dépend de leur meilleure connaissance par ceux, «élus, maîtres d'ouvrage, concepteurs, [qui] commandent et produisent des œuvres urbaines architecturales et paysagères en cohérence avec le milieu dans lequel elles s'insèrent, avec les capacités économiques locales et aux services des habitants auxquelles elles s'adressent » (ibidem, p. 6), est tenace. Le discours d'appel à rompre avec les modèles d'urbanisme antérieurs et à opter pour le « renouvellement urbain » ne manque pas, mais est-il suivi d'effet?

Pour approfondir et préciser cette hypothèse du faible renouvellement des pratiques scientifiques par la référence au développement durable, l'analyse de la littérature récente produite par les chercheurs des sciences urbaines (en particulier de géographie) a été faite au filtre d'un certain nombre de questions : considèrentils que la notion de "développement durable urbain » n'est qu'un recyclage ou un replâtrage des utopies et de politiques antérieures (autour des notions de compacité, d'étalement urbain, $\mathrm{d}^{\prime}$ « automobilisation »...) et que la reproduction/régulation du fait urbain reste la perspective et la pratique dominantes? Y a-t-il un véritable investissement scientifique portant sur les sens de la notion nouvelle et sur sa capacité à faire émerger de nouveaux paradigmes? Ou, là encore, ne s'agit-il que

(Jollivet, 1992). De ce travail, est né l'objectif de monter en généralité pour concevoir et mettre en œuvre une pratique de recherche intégrant systèmes naturels et systèmes sociaux. Ici, en l'occurrence, dans les «systèmes » urbains.

${ }^{6}$ Cette montée en généralité, notamment à travers la modélisation, est précisément l'objectif de la revue généraliste Natures Sciences Sociétés.

7 De «Quelle ville pour demain? » (PUCA, 1998) jusqu'à la récente université d'été de la DIACT «Où vont les villes?» (Lille, 20-21 septembre 2006). 
de la simple reproduction aménagée de points de vue essentiellement disciplinaires ? Quelle place est accordée à la dimension « matérielle » (« naturelle», « environnementale », de «biodiversité ») du développement urbain durable dans la mesure où ce qui fait partie de la «nature » en ville se limite généralement à la «belle nature » (les espaces verts) ou à une idée très stéréotypée des ressources (l'air, l'eau, les déchets...), mal mise en rapport et avec le territoire urbain proprement dit et avec les autres dimensions du développement durable?

$\mathrm{Au}$ terme de cette analyse, quatre types d'attitude peuvent être distingués parmi les chercheurs confrontés à l'utopie politique du développement durable (Mathieu et Guermond, 2005b ; Mathieu, 2006). La première, particulièrement sensible chez les tenants de l'analyse spatiale, est le rejet de la notion elle-même, parce que trop chargée d'idéologie, trop ancrée dans l'écologisme, voire le biologisme (Pelletier, 1993), ou même porteuse de risque d'aggravations des inégalités sociales (Brunet, 1998). La deuxième se caractérise par l'indifférence à la nouvelle idéologie, désintérêt qui se rencontre couramment lorsque la spécialisation thématique ou l'identification à un courant théorique bien affirmé (par exemple, la géographie politique ou culturelle ou la sociologie du travail) sont privilégiées, rendant alors insensible à l'idée même de complexité qu'implique la notion de développement durable.

Mais j'ai montré que la troisième attitude : « une adoption de la référence au développement durable qui va de soi, ne bouleversant ni les concepts ni les pratiques scientifiques antérieures »(Mathieu, 2006), est aujourd'hui dominante, tant dans les sciences sociales urbaines que chez les ruralistes. Il ne s'agit plus de l'indifférence des premiers temps, mais d'un opportunisme généralisé, qui consiste le plus souvent en un recyclage des théories et des idées courantes dans le domaine pratiqué. Le qualificatif de «durable » devient alors «la » norme scientifique incontournable, en somme, une addition de «poudre » rhétorique habillant des préoccupations et des trajectoires scientifiques qui restent en fin de compte inchangées.

Au contraire, «prendre l'utopie politique à la lettre pour produire un nouveau mode de connaissance » résume donc une quatrième attitude à laquelle je me rattache et que la multiplication des discours scientifiques sur le thème $\mathrm{m}$ 'incite à formaliser (ibidem, 2006). Comme l'écrit Marcel Jollivet (2001, p. 115), l' « idée de développement durable est une puissante invitation à un exercice d'auto-réflexivité que l'humanité s'adresse à elle-même sur la base de ses expériences passées ». Il s'agit donc ici d'énoncer le plus clairement possible la position théorique "radicale » que j'adopte : l'appel à une discontinuité, à une rupture dans le travail scientifique, sur la base de la question que pose le passage de l'utopie politique du développement durable à sa réalisation au sens propre. Parce qu'il s'agit d'un appel à faire de l'utopie politique du développement durable un «événement scientifique », la forme du manifeste s'est imposée, ainsi que le titre qui lui est donné.

Je mets donc en discussion quelques-uns des principes qui, selon moi, sont les conditions de cette radicalité scientifique nécessaire pour penser la durabilité urbaine, en espérant qu'ils ouvriront un large débat.

\section{L'acceptation de l'essence utopique du développement (urbain) durable}

En premier lieu, il s'agit de prendre toute la mesure du moment historique dans lequel est mobilisée la notion de développement durable (Jollivet, 2001). Depuis la fin des années 1990, qui marque en quelque sorte le début de son introduction, la période actuelle constitue le «moment » de plein essor d'une idéologie politique naissante dont le succès tient au fait qu'elle produit un sentiment d'évidence : l'effectivité probable, voire vraisemblable, qu'une utopie se réalise au sens propre, s'ancre dans des lieux. En effet, se diffuse, dans la sphère politique et dans l'opinion, l'idée qu'une sortie de crise est possible parce que la mise en avant de l'idée d'un développement possiblement durable introduit celle d'un renversement des pratiques politiques et celle de l'entrée dans une période " nouvelle », dans laquelle des solutions pourraient être trouvées à des problèmes soit récurrents (la pauvreté), soit perçus de façon plus aiguë (la question de l'environnement), et à des contradictions quasiment insolubles dans les formes de développement héritées de la période précédente.

En somme, le moment actuel est celui de l'irruption d'une utopie politique dont le destin est de devenir une idéologie dominante, id est qui a manifestement vocation à envelopper (plus même, à englober) l'ensemble des discours : au premier chef, ceux de la sphère politique qui prétend définir les orientations prioritaires de l'action, mais aussi, en interaction, ceux de la sphère scientifique qui, souvent suivistes, accompagnent ou légitiment les formes de l'action politique. De mon point de vue, ce «moment » est semblable à la période qui vit naître l'Aménagement du territoire. Née aux lendemains de la seconde guerre mondiale, cette idée concentrait également tous les ingrédients pour se présenter comme une invention politique forte, notamment du point de vue du champ d'action de l'État. Apparaissant comme un outil susceptible non seulement d'annuler les destructions de la guerre, mais de construire les bases d'un monde nouveau, équilibrant l'économique et le social par la «justice territoriale », elle peut rétrospectivement être également qualifiée d'utopie politique. Et elle a été pendant au moins deux décennies la référence majeure pour de nombreuses disciplines scientifiques. 
Or, il me semble essentiel de prendre acte de ce que ces deux moments se ressemblent pour ne pas reproduire, dans ce temps des origines du développement durable, les attitudes scientifiques «soumises » ou « opportunistes » qui risquent d'accompagner l'irruption d'une utopie politique qui se popularise aisément, comme ce fut le cas avec la vogue de la référence à l'aménagement du territoire.

Le propre du scientifique est en effet de définir une autonomie de pensée face à des idéologies de cette ampleur et de ne pas abandonner aux politiques seuls (et aux techniciens) la légitimité de dire ce qu'il faut faire et comment faire, puis de se contenter d'analyser les politiques en train de se faire. C'est la raison pour laquelle, tout en reconnaissant la qualité des travaux qui sont faits dans cet esprit, je considère comme une réponse scientifique « insuffisante» (voire "faible») les recherches sur la ville durable qui se limitent à l'identification des cas exemplaires, les «quartiers durables » qui seraient déjà le modèle à diffuser pour atteindre "la durabilité » de l'ensemble des milieux urbains.

Je pars du principe que l'acceptation du caractère utopique du développement urbain durable est le seul moyen de construire cette autonomie scientifique nécessaire pour produire les connaissances utiles à une action politique (à un accompagnement du changement social) s'approchant le plus possible de la réalisation de l'utopie proprement dite. Il faut en effet prendre acte du fait que la notion de développement durable urbain qui s'érige en catégorie de pensée et d'action ne peut être opératoire si elle n'est pas d'emblée considérée comme « irréalisable », impossible à atteindre dans le réel du fait de sa complexité et surtout du caractère contradictoire des objectifs qu'elle prétend concilier. Se donner comme objectif de recherche de résoudre les incompatibilités entre développement économique, préservation des ressources et des biens patrimoniaux, équité sociale, pour un même territoire, et pour le long terme, n'est pensable que dans la conscience de son caractère utopique et donc de l'impossibilité de l'atteindre dans ces termes. Ici encore, la référence à Sartre est intéressante et, même, s'impose. Elle renvoie pour le coup à son ouvrage Les Carnets de la drôle de guerre : «La volonté a besoin du monde et de la résistance des choses [...]. Seule en effet, la résistance d'un réel permet de distinguer ce qui est possible de ce qui est, et de projeter par-delà de ce qui est le possible ${ }^{8}$.» Telle est

\footnotetext{
${ }^{8}$ Cité par Gérard Wormser (2005, p. 17). Pour ce philosophe, les recherches où «Sartre se tourne vers la compréhension de situations singulières [...] restent sous-estimées dans leur portée critique à l'égard des sciences sociales. Elles interrogent les fonctions de la connaissance et des institutions dans une société développée : Sartre fait des sciences sociales la médiation pour accéder à une vérité humaine, comme en témoigne la création des Temps modernes, cette réflexion collective à partir de la phénoménologie».
}

bien la seconde obligation d'une démarche scientifique prenant acte des exigences que le concept de développement durable implique.

\section{Le choix de la complexité : partir des objectifs les plus difficiles à concilier pour construire le problème de développement urbain durable comme objet de recherche}

Dès lors, l'acceptation du développement urbain durable comme une utopie implique de définir l'objet de recherche à partir des deux "piliers » du développent durable qui me semblent les plus difficiles à concevoir et à intégrer dans une politique quand il s'agit d'urbanisme et d'espace urbain?.

Le premier pilier est celui de la gestion durable des ressources dans la ville - et plus spécifiquement des ressources dites naturelles. Une question est alors essentielle à clarifier : qu'est-ce que la nature (l'environnement) en ville et pourquoi son "effacement " (Blanc et Mathieu, 1996) ? Comment dépasser son assimilation au végétal et à une biodiversité de décor, à la «belle nature » sans relation autre que paysagère et symbolique avec l'idée de durabilité (Calenge, 1997)? L'approfondissement de ce que sont les "natures » en milieu urbain (Hucy et al., 2001,2005 ) est une priorité scientifique si l'on cherche à sortir des stéréotypes pour penser la dimension naturelle et environnementale de la durabilité urbaine.

L'idée que ville et nature sont des concepts antagoniques - le milieu urbain (technique) ne pouvant être qu'artificiel, la nature en ville ne pouvant être qu'importée et voulue - a produit une réelle difficulté à penser la signification du terme de ressource appliqué à l'espace urbain. Qu'est-ce qu'une «ressource urbaine »? Comment penser les «natures de la ville» comme des bases de production de ressources? Autant de concepts nouveaux à instruire. Faut-il se contenter de penser que les ressources «naturelles » sont hors la ville et que les gérer en ville, c'est se limiter à l'observation et au contrôle de leur usage? Ou bien faut-il penser la matérialité même des espaces urbains comme des ressources sinon naturelles, du moins matérielles et, de fait, en relation avec les phénomènes naturels? Repenser ce qui fait nature et ce qui fait ressource dans les milieux urbains, c'est repositionner les questions de l'air, de l'eau et, d'une façon

\footnotetext{
${ }^{9}$ Ce n'est pas que le «pilier économique» ne pose pas de problèmes, mais les exigences qu'il soulève sont le plus souvent considérées comme prioritaires dans la sphère des acteurs politiques et des projets de développement, d'où aussi la suprématie de la science économique sur la pensée politique du développement durable. L'objectif de ce texte est de rééquilibrer les approches et de contribuer à ouvrir la voie à une articulation des deux autres piliers.
} 
générale, de ce qu'on appelle les "pollutions » urbaines dans des processus naturels dont elles font partie.

Mais, même si elle est apparemment mieux connue car très investie par les sciences sociales ${ }^{10}$, l'approche scientifique de la dimension sociale, second pilier de l'utopie du développement durable, est également à construire dans une radicalité et de façon spécifique. Car il ne s'agit pas seulement de mettre au jour les inégalités sociales et leur rapport avec les territoires de la ville, voire de rajeunir ce concept en lui substituant celui d'inégalités écologiques. Il s'agit de réinventer les concepts et les démarches qui permettent d'établir les liens entre pauvreté, équité sociale et gestion durable des ressources dans un milieu urbain donné. La question de fond est : que faut-il entendre hic et nunc par «milieu de vie socialement durable » ? Mon hypothèse est qu'il faut tenter $d^{\prime} y$ répondre en mettant l'habitant et le concept de mode d'habiter au centre de l'analyse sociale (Hucy et al., 2005). Qu'est-ce qui est constitutif du «bien-être» de chaque habitant dans son rapport avec les «valeurs des lieux » ? Ceci oblige à tenir compte simultanément de tous les milieux de vie de l'habitant $^{11}$ dans ses différentes pratiques des lieux. Comment peuvent être conciliées ses représentations et ses pratiques en tous ses lieux de vie et la préservation d'un bien collectif, si ce n'est commun, à savoir les ressources non renouvelables et la biodiversité des milieux urbains?

C'est le défi que le problème de la conciliation des deux objectifs particulièrement exclusifs - voire contradictoires - en milieu urbain pose qui pourrait permettre de définir les nouveaux objets de recherche ouverts par l'utopie du développement (urbain) durable. Car qu'estce qu'un "problème de développement durable urbain", si ce n'est l'analyse - au sens fort - d'une " situation ${ }^{12}$ » dans laquelle articuler la dimension environnementale et la dimension sociale d'un système spatial urbain pose problème. Dans sa traduction politique, un problème de développement urbain durable est une «situation » complexe : on doit connaître tous les processus qui sont susceptibles d'y interférer dans une perspective d'une meilleure gouvernance, $d^{\prime}$ un meilleur équilibre entre les trois piliers du développement durable. L'« habitabilité durable » des (mi)lieux urbains, comme problème de développement urbain durable, pourrait être le fil directeur pour analyser les «situations » urbaines. C'est pourquoi cette analyse rend nécessaire de repenser toutes les «natures » de la ville dans leur interaction avec les représentations et les pratiques sociales qui les mettent en jeu. Elle exige aussi de repenser ce que recouvre la notion de sociétés locales urbaines en redonnant une place à l'habitant,

\footnotetext{
${ }^{10}$ Le thème des inégalités sociales est très présent dans les sciences de la ville, les concepts de « ségrégation sociale et spatiale », de «communautés fermées » en témoignent.

11 On pourrait parler de l'« habitant total » au sens de Mauss.

$12 \mathrm{Au}$ sens sartrien.
}

à la personne habitante (avec sa subjectivité, sa sensibilité, sa culture de la nature et ses pratiques sur le milieu naturel...) en tant qu'elle est un des lieux d'articulation essentiels des dimensions environnementales et sociales de la durabilité (Mathieu, 2000a). On pourrait faire l'hypothèse que la question économique, le troisième pilier, n'aurait à être pris en compte et à être évalué qu'une fois faite l'analyse des interrelations entre équité sociale et préservation des ressources et de la biodiversité urbaines.

\section{Mobiliser les acquis des pratiques interdisciplinaires pour comprendre et modéliser les interrelations sociétés/natures en milieu urbain}

On en vient à la démarche proprement dite requise pour atteindre l'objectif annoncé au départ : produire un mode de connaissance spécifique pour aller vers la «mise en réalité » du développement urbain durable, c'est-àdire vers une réelle prise en charge de ses prérequis.

Il importe tout d'abord d'énoncer la posture théorique à laquelle la méthode doit correspondre pour se conformer aux principes énoncés. Instruire sérieusement la question de la durabilité des villes et des quartiers urbains et définir une politique en la matière exige en effet un renversement de la démarche, encore la plupart du temps descendante, à laquelle on se réfère lorsqu' on parle du développement durable. L'incitation à la démocratie participative va dans ce sens, mais il manque précisément les connaissances indispensables pour qu'elle puisse être mise en pratique de façon rigoureuse. Il faut revenir à la question posée plus haut : qu'est-ce que la durabilité (l'habitabilité durable) d'un milieu urbain? Répondre à cette question suppose que l'on connaisse le point de vue des habitants sur leurs milieux de vie. C'est en partant de là que $\mathrm{l}^{\prime}$ on se donnera les moyens $\mathrm{d}$ 'instruire le politique par le scientifique au lieu de l'accompagner (même sous forme critique) par un suivi scientifique.

Ce renversement opéré, quatre conditions doivent être réalisées : l'interdisciplinarité; le croisement territorialisé des données; l'inventivité méthodologique; le recours à un concept intégrateur : le «mode d'habiter».

Le respect de la première condition passe par la construction d'un objet mixte - un macro-objet (Robic et Mathieu, 2001) -, ayant la propriété d'être «urbain », dans lequel les dimensions naturalistes (matérielles) et les dimensions sociales (représentations/pratiques, conflits/pouvoirs, appropriation/exclusion) ne peuvent pas être dissociées dans la pratique de recherche. Cet objet mixte ne peut pas être appréhendé par une simple «interdisciplinarité de proximité», mais requiert une «interdisciplinarité élargie » associant sciences de la vie et sciences de la société (Jollivet et Legay, 2005). 
Il faut donc, seconde condition, utiliser une méthodologie permettant de confronter des données de natures et de temporalités intrinsèquement différentes. Cela conduit à privilégier une approche par « sites », voire par «microsites» (Hucy et al., 2005; Blanc et al., 2005). Ces sites doivent être des lieux communs à toutes les disciplines qui consentent à $\mathrm{y}$ traiter du problème dans une formulation partagée ayant force de contrat. La mise en relation constante des problématiques, des données recueillies, des hypothèses d'interaction, doit se faire sur des espaces pertinents définis au terme de discussions entre les chercheurs et dans lesquels s'effectue un travail parallèle mais toujours colocalisé. D'où le recours à une modélisation territorialisée et la nécessité d'articuler cette nouvelle démarche à celle de l'analyse spatiale (Mathieu et Guermond, 2005).

De ces deux premières conditions découle la nécessité de la troisième, à savoir une mise à plat des théories et des méthodes habituelles et l'exploration, voire l'invention, de concepts rendant compte des interactions entre processus et champs scientifiques hétérogènes. Certes, on pourra se référer aux acquis des pratiques interdisciplinaires qui se sont souvent appliquées aux problématiques rurales (par exemple, Cohen et Duqué, 2001; Cohen, 2003). Mais l'essentiel est d'inventer les pratiques scientifiques les plus adéquates.

Enfin - et c'est sans doute la condition la plus proche de «l'intentionnalité morale » sartrienne -, il faut mettre l'habitant et le concept de «mode d'habiter » au centre de la démarche d'analyse proprement dite (Mathieu, 2000b). C'est en effet en écoutant la parole de l'habitant que le chercheur donne à son travail une dimension politique. Il faut oser s'engager dans une anthropologie interdisciplinaire de l'habitant et du mode d'habiter, une interdisciplinarité anthropologique en quelque sorte, appliquée aux sociétés postindustrielles. En évitant les concepts surplombants, comme celui de médiance, ou une confiance trop grande accordée aux philosophies occidentales pour rendre compte de la relation culture/nature, il faut écouter et approfondir la parole des habitants pour aller jusqu'à la compréhension de leurs rapports à la nature et à leurs milieux de vie. Recueillir patiemment des « récits de lieux de vie » (Mathieu et al., 2004), faire l'analyse des pratiques habitantes jardinières, des rapports à l'animal désiré et non désiré, ceci dans tous les lieux de vie en ville et dans la maison de campagne ou les lieux rêvés de vacances... devrait conduire à mettre au jour les catégories à travers lesquelles chaque habitant pense son rapport aux lieux, aux natures, au mode d'habiter dans tous les sens du terme (se loger, circuler, travailler, vivre ensemble). Comme le montrent déjà ces premières expériences d'anthropologie interdisciplinaire (Hucy et al., 2005 ; Grésillon, 2004, 2005), l'identification des pratiques et des représentations de citadins, le repérage de l'écart entre le rêvé et le réel, en particulier autour des catégories de «dedans/dehors », de «maison (chez soi)/hors de chez soi », est une voie pour aller au fond de la question de ce qu'est la durabilité du point de vue des consciences « ordinaires» et des logiques habitantes.

En conclusion, il me faut revenir sur le sens de ce manifeste. S'interroger sur la durabilité urbaine suppose une prise en compte systémique de la gestion des villes comme de nombreux travaux le font. Mais la marche vers l'utopie du développement durable ne pourra pas se faire sans une reconnaissance et une activation accrue du rôle des habitants dans la «fabrique » de la ville et des milieux urbains. C'est cet enjeu qui justifie la référence à Sartre prise comme point de départ. Ce texte est un appel à ne pas prendre les chemins déjà bien tracés qui associent les chercheurs aux politiques : ceux qui savent ce qu'est la biodiversité et ce que veut dire ville durable étant, de fait et de droit, les conseillers du prince. Et à ne pas prendre non plus les itinéraires qui commencent par des modèles politiques déjà élaborés qui sont supposés mener, sans connaissances scientifiques supplémentaires, jusqu'au citoyen. Ces deux démarches écartent quelque chose qui est décisif : le potentiel de changement et de responsabilité que constitue l'habitant. Le prendre en compte, c'est se donner une opportunité de rééquilibrer l'empreinte écologique de chacun dans ses lieux de vie. Cela conduit à s'interroger sur la notion de participation citoyenne, à la fois sur un plan politique et sur un plan scientifique. En somme, ce texte est une invitation pour le chercheur à s'engager autrement, à partir du politique au sens fort - en l'occurrence de l'utopie politique du développement durable-, pour viser des actes scientifiques libres et autonomes, tournés vers le futur et tendus vers l'impossible pour tenter de les atteindre. Ceci ne récuse pas les recherches faites dans les champs des sciences de la nature, mais pose la question de leur relation au politique. Car l'objectif est bel et bien de réussir ce passage du politique au scientifique et réciproquement. C'est un appel à un changement des pratiques scientifiques pour leur redonner un sens dans le politique, ou plutôt dans l'invention du politique proprement dit.

\section{Remerciements}

Je remercie Dominique Couret et Marcel Jollivet pour la pertinence et la sympathie de leur relecture qui m'a aidée à aller jusqu'au bout de ma pensée et de mon écriture.

\section{Références}

Blanc, N., Mathieu, N., 1996. Repenser l'effacement de la nature dans la ville, Le Courrier du CNRS, 82, 105-107.

Blanc, N., Bridier, S., Glatron, S., Grésillon, L., Cohen, M., 2005, Appréhender la ville comme (mi)lieu de vie. L'apport d'un dispositif interdisciplinaire de recherche, in Mathieu N., Guermond, Y. (Eds), La Ville durable, du politique au scientifique, Paris, Cemagref/Cirad/Ifremer/Inra, 261-281. 
Brunet, R., 1998. Le développement durable en haut de l'échelle, Pouvoirs locaux, 34.

Calenge, C., 1997. De la nature de la ville, Annales de la recherche urbaine, 74, 12-19.

Cohen, M. (Ed.), 2003. La Brousse et le berger : une approche interdisciplinaire de l'embroussaillement des parcours, Paris, CNRS Éditions.

Cohen, M., Duqué, G., 2001. Les Deux Visages du Sertão : stratégies paysannes face aux sécheresses, Paris, IRD Éditions.

Godard, O., 2001. Le développement durable et la recherche scientifique, ou la difficile conciliation des logiques de l'action et de la connaissance, in Jollivet, M. (Ed.), Le Développement durable, de l'utopie au concept : de nouveaux chantiers pour la recherche, Paris, Elsevier, 61-81.

Dubois-Taine, G. (Ed.), 1998. La Ville émergente : constats pour renouveler les lignes d'action publiques, Paris, PUCA.

Grésillon, L., 2004. La ville durable ou l'urbanisme au risque du bien-être sensoriel, Développement durable et Territoires, dossier 4 : «La ville et l'enjeu du développement durable».

Grésillon, L., 2005. Sentir Paris, bien-être et valeurs des lieux. Thèse de doctorat, Université de Paris 1.

Hucy, W., Mathieu, N., Freire-Diaz, S., 2001. Nature in town: what criteria to introduce in the GIS of Rouen - La nature dans la ville. Quels critères introduire dans le SIG de Rouen?, Cybergeo, 202

(http://www.cybergeo.presse.fr/durham/hucy/hucy.htm).

Hucy, W., Mathieu, N., Mazellier, T., Raynaud, H., 2005. L'habitabilité des milieux urbains : un objet au croisement des disciplines, in Mathieu, N., Guermond, Y. (Eds), La Ville durable, du politique au scientifique, Paris, Cemagref/Cirad/Ifremer/Inra, 237-260.

Jollivet, M., 1992. Sciences de la nature, sciences de la société : les passeurs de frontières, Paris, CNRS Éditions.

Jollivet, M. (Ed.), 2001. Le Développement durable, de l'utopie au concept : de nouveaux chantiers pour la recherche, Paris, Elsevier.

Jollivet, M., Legay, J.-M., 2005. Canevas pour une réflexion sur une interdisciplinarité entre sciences de la nature et sciences sociales, Natures Sciences Sociétés, 13, 2, 184-188.
Legay, J.-M., Schmid, A.-F., 2004. Philosophie de l'interdisciplinarité : correspondance (1999-2004) sur la recherche scientifique, la modélisation et les objets complexes, Paris, Pétra.

Mathieu, N., 2000a. Repenser la nature dans la ville : un enjeu pour la géographie, Natures Sciences Sociétés, 8, 3, 74-82.

Mathieu, N., 2000b. Des représentations et pratiques de la nature aux cultures de la nature chez les citadins : question générale et étude de cas. BAGF, 2, 162-174.

Mathieu, N., 2006. La géographie rurale française face à l'utopie du développement durable : quelles réactions, quelles perspectives?, Boletín de la Asociación de Geógrafos Españoles, 41, 39-68.

Mathieu, N., Jollivet, M. (Eds), 1989. Du rural à l'environnement: la question de la nature aujourd'hui, Paris, ARF/L'Harmattan.

Mathieu, N., Morel-Brochet, A., Blanc, N., Gajewski, P., Grésillon, L., Hebert, F., Hucy, W., Raymond, R., 2004. Habiter le dedans et le dehors : la maison ou l'Eden rêvé et recréé, STRATES, 11, 267-288.

Mathieu, N., Cohen, M., 2005. Vers une construction interdisciplinaire du concept de milieu (urbain) durable, in Actes du colloque de l'Observatoire universitaire de la ville et du développement durable "Développement urbain durable, gestion des ressources et gouvernance », Université de Lausanne, 21-23 septembre 2005, Lausanne, Unil (DVD).

Mathieu, N., Guermond, Y., 2005a. La Ville durable, du politique au scientifique, Paris, Cemagref/Cirad/Ifremer/Inra.

Mathieu, N., Guermond, Y., 2005b. Introduction. La ville durable : un enjeu scientifique, in Mathieu, N., Guermond, Y. (Eds), La Ville durable, du politique au scientifique, Paris, Cemagref/Cirad/Ifremer/Inra, 11-29.

Pelletier, P., 1993. L'Imposture écologique, Montpellier, Maison de la Géographie/GIP RECLUS.

Robic, M.-C., Mathieu, N., 2001, Géographie et durabilité : redéployer une expérience et mobiliser de nouveaux savoirfaire, in Jollivet, M. (Ed.), Le Développement durable, de l'utopie au concept : de nouveaux chantiers pour la recherche, Paris, Elsevier, 167-190.

Wormser, G., 2005. Sartre, du mythe à l'histoire, Sens public, 3-4, $15-46$.

To access this journal online: www.edpsciences.org 